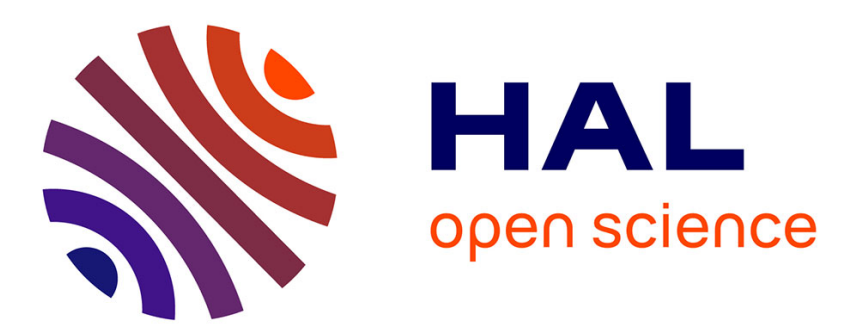

\title{
Improving DTI Resolution from a Single Clinical Acquisition: A Statistical Approach using Spatial Prior
}

\author{
Vikash Gupta, Nicholas Ayache, Xavier Pennec
}

\section{To cite this version:}

Vikash Gupta, Nicholas Ayache, Xavier Pennec. Improving DTI Resolution from a Single Clinical Acquisition: A Statistical Approach using Spatial Prior. Proceedings of Medical Image Computing and Computer Assisted Intervention 2013 (MICCAI), Sep 2013, Nagoya, Japan. pp.477-484, 10.1007/9783-642-40760-4_60. hal-00845927

\section{HAL Id: hal-00845927 https://hal.inria.fr/hal-00845927}

Submitted on 18 Jul 2013

HAL is a multi-disciplinary open access archive for the deposit and dissemination of scientific research documents, whether they are published or not. The documents may come from teaching and research institutions in France or abroad, or from public or private research centers.
L'archive ouverte pluridisciplinaire HAL, est destinée au dépôt et à la diffusion de documents scientifiques de niveau recherche, publiés ou non, émanant des établissements d'enseignement et de recherche français ou étrangers, des laboratoires publics ou privés. 


\title{
Improving DTI Resolution from a Single Clinical Acquisition: A Statistical Approach using Spatial Prior
}

\author{
Vikash Gupta, Nicholas Ayache, and Xavier Pennec \\ INRIA Sophia Antipolis - ASCLEPIOS Project
}

\begin{abstract}
Diffusion Tensor Imaging (DTI) provides us with valuable information about the white matter fibers and their arrangement in the brain. However, clinical DTI acquisitions are often low resolution, causing partial volume effects. In this paper, we propose a new high resolution tensor estimation method. This method makes use of the spatial correlation between neighboring voxels. Unlike some super-resolution algorithms, the proposed method does not require multiple acquisitions, thus it is better suited for clinical situations. The method relies on a maximum likelihood strategy for tensor estimation to optimally account for the noise and an anisotropic regularization prior to promote smoothness in homogeneous areas while respecting the edges. To the best of our knowledge, this is the first method to produce high resolution tensor images from a single low resolution acquisition. We demonstrate the efficiency of the method on synthetic low-resolution data and real clinical data. The results show statistically significant improvements in fiber tractography.
\end{abstract}

Keywords: DTI, Super-resolution, Partial volume effect

\section{Low Spatial Resolution in clinical DTI}

DTI is a non-invasive imaging modality used for mapping the diffusion of water molecules in the brain. The diffusion process depends on the tissue microstructure and thus makes imaging white matter (WM) fibers and their organization in the brain feasible. It can be used to study the brain connectivity through a tractography analysis. In the clinics, DTI is used to study WM disorders. However, clinical DTI acquisitions have usually low spatial resolution (typically 2.0 - 5.5 $\mathrm{mm}$ in each direction). In practice, it is often desirable to visualize and gather fiber structure information from high resolution (HR) images. But, acquiring HR diffusion weighted images (DWI) requires either longer acquisition time or using scanners with stronger magnetic fields $(7 \mathrm{~T}$ and $11 \mathrm{~T})$, compared to the ones used in a clinical setting $(1.5 \mathrm{~T}$ and $3.0 \mathrm{~T})$. Both of these options are not suitable for clinical scenarios. So, resampling the low resolution DTI to a high resolution space is currently the only option available to clinicians. However, the present resampling techniques do not account for partial volume (PV) effects present 
in the clinical images at native resolution. The PV effects in the DWI leads to an underestimation of tensors adversely affecting fiber tractography [1]. In this work, we propose a tensor estimation method, which takes into account the partial volume effect and yields a more spatially coherent tensor field. The efficacy of the method is established through significant improvements in tractography.

\section{Super-resolution reconstruction}

One of the first super-resolution algorithms for MRI proposed by Peled et al. uses a combination of spatially shifted single shot diffusion weighted images to create HR images [2]. This method uses eight repeated low resolution scans with a shifted field of view and different b-values each time. In another study [3], the volume acquisition is spatially shifted in the slice-selected direction and inter slice reconstruction is achieved using the Irani-Peleg's [4] back-projection method. But this method was not extended for DTI studies. More recently, super-resolution on diffusion weighted images using multiple anisotropic orthogonal DWI scan has been proposed [5]. All these methods of super-resolution rely on multiple acquisitions of the same subject. While this may be an acceptable practice in a research environment, this would be difficult and even undesirable in a clinical setting. Unlike previous methods, we propose a HR tensor reconstruction algorithm which does not require multiple acquisitions. Our method also accounts for the PV in the low resolution DWIs, producing DTI at higher resolutions. The proposed method can be related to the regularized super-resolution method of [6], which recovers WM fiber configuration based on volume fraction of anisotropic fiber population in the neighboring voxels. However, we use anisotropic regularization to recover the full tensor information. The method is described in section 2. Experiments performed on simulated low resolution acquisitions in section 3.1 show that the fractional anisotropy (FA) image computed using HR tensor estimation is better contrasted and less blurry compared to the one computed using tensor resampling. A comparison of FA images on real data and quantitative evaluation on fiber tractogaphy is presented in sections 3.2 and 3.3 respectively.

\section{Statistical Tensor Reconstruction with spatial prior at any resolution}

The diffusion tensor $D$ is related to each DWI $S_{i}$ corresponding to the encoding gradient $g_{i}$ and the image with null gradient $S_{0}$ using the Stejskal-Tanner Equation [7], $S_{i}=S_{0} e^{-b g_{i}{ }^{T} D g_{i}}$ where $b$ is the diffusion factor. $D$ is a second order tensor (i.e., a symmetric positive definite matrix). We build our tensor estimation model based on variational methods for joint estimation and smoothing of DTI [8] in the Log-Euclidean framework. 


\subsection{A discrete signal degradation model: From high resolution to low resolution images}

A voxel in the low resolution (LR) image $x_{k}$ is composed of the voxels $y_{j}$ in the HR image. The observed signal $S^{L R}\left(x_{k}\right)$ in the voxel $x_{k}$ is the weighted sum of the intensities of the voxels $y_{j}$ :

$$
S^{L R}\left(x_{k}\right)=\sum_{j} \alpha_{k j} S^{H R}\left(y_{j}\right)
$$

where $S^{H R}\left(y_{j}\right)$ is the signal intensity in the voxel $y_{j}$ of the HR image; $\alpha_{k j}$ is the partial volume overlap of the HR voxel $y_{j}$ in the LR voxel $x_{k}$. Based on the above model, each LR gradient image, $S_{i}^{L R}$ can be written in terms of the underlying $\mathrm{HR}$ tensors $\left(D^{H R}\right)$ and the $S_{0}^{H R}$ (image with null gradient in high resolution):

$$
S_{i}^{L R}\left(x_{k}\right)=\sum_{j} \alpha_{k j} S_{0}^{H R}\left(y_{j}\right) e^{-b g_{i}{ }^{T} D^{H R}\left(y_{j}\right) g_{i}} .
$$

For simplicity, we write $S_{i}^{L R}$ as $S_{i}$ and expand the term using Equation 1, when necessary.

\subsection{Tensor Estimation}

With the above image degradation model, we solve the inverse problem of estimating tensors in the HR space. This is an ill-posed inverse problem because for a given signal $S_{i}\left(x_{k}\right)$ there is an infinite number of combinations of $D\left(y_{j}\right)$ possible in Equation 1. In the Log-Euclidean framework, we define L as the matrix logarithm of D: $\mathrm{L}=\log (\mathrm{D})$. Following [8], the tensor estimation can be looked upon as a variational formulation, i.e., one should minimize the following energy functional,

$$
\mathrm{E}\left(\mathrm{S}_{0}, \mathrm{~L}\right)=\frac{1}{2} \operatorname{Sim}\left(\mathrm{S}_{0}, \mathrm{~L}\right)+\frac{\lambda_{\mathrm{s}}}{2} \operatorname{Reg}\left(\mathrm{S}_{0}\right)+\frac{\lambda_{\mathrm{L}}}{2} \operatorname{Reg}(\mathrm{L}),
$$

where $\operatorname{Sim}($.$) is the data fidelity term and \operatorname{Reg}\left(\mathrm{S}_{0}\right), \operatorname{Reg}(\mathrm{L})$ are the regularization priors on $\mathrm{S}_{0}$ and $\mathrm{L}$ images with the respective weights $\lambda_{s}$ and $\lambda_{L}$. In [8], the tensor estimation is performed at the same resolution as that of acquisition. However, in this paper, we aim to estimate tensors at a resolution higher than the acquisition resolution. The LR acquired signal and the HR estimated tensors are linked through the degradation model described in Equation 1. Thus, the solution of the energy minimization problem is an optimal tensor field, which is spatially coherent and is consistent with the observed signal.

\subsection{Similarity Criteria for Tensor Estimation}

The observed signal in any DWI can be modeled as $\tilde{S}_{i}=S_{i}+\eta$, where $\tilde{S}_{i}$ is the observed DWI, $S_{i}$ is the true image (as in Equation 1) and $\eta$ is the associated 
noise model. As described in [8], the data attachment term $\mathrm{E}($.$) corresponds to$ a maximum likelihood estimator (MLE) adapted to the noise model. The MLE for the probability density function of a given noise model is,

$$
\operatorname{Sim}_{M L}(.)=-\sum_{i=0}^{N} \sum_{x_{k}} \log \left[p\left(\tilde{S}_{i}\left(x_{k}\right) \mid S_{i}\left(x_{k}\right)\right)\right]
$$

where $S_{i}\left(x_{k}\right)$ is the same as in Equation 1 and $p($.$) is the probability density$ function (pdf) of the the noise model. Unlike [8], $E($.$) is a function of two vari-$ ables, the HR image with null gradient, $S_{0}\left(y_{j}\right)$ and the logrithm of HR tensor field, $L\left(y_{j}\right)$. The maximization is achieved using the steepest descent algorithm. The gradient of $\operatorname{Sim}_{M L}\left(S_{0}\left(y_{j}\right), L\left(y_{j}\right)\right)$ by differentiating Equation 3 is,

$$
\nabla \operatorname{Sim}_{M L}=\left[\begin{array}{ll}
\frac{\partial \operatorname{Sim}_{M L}}{\partial S_{0}\left(y_{j}\right)} & \frac{\partial \operatorname{Sim}_{M L}}{\partial L\left(y_{j}\right)}
\end{array}\right]^{T}
$$

Taking partial derivatives of Equation 3 with respect to $S_{0}\left(y_{j}\right)$ and $L\left(y_{j}\right)$,

$$
\frac{\partial \operatorname{Sim}_{M L}}{\partial S_{0}\left(y_{j}\right)}=-\sum_{i=0}^{N} \sum_{x_{k}} r(.) \frac{\partial \tilde{S}_{i}\left(x_{k}\right)}{\partial S_{0}\left(y_{j}\right)} ; \quad \frac{\partial \operatorname{Sim}_{M L}}{\partial L\left(y_{j}\right)}=-\sum_{i=0}^{N} \sum_{x_{k}} r(.) \frac{\partial \tilde{S}_{i}\left(x_{k}\right)}{\partial L\left(y_{j}\right)}
$$

where $r()=.p^{\prime}(.) / p($.$) and p^{\prime}($.$) is the derivative of p($.$) with respect to \tilde{S}_{i}\left(x_{k}\right)$. Differentiating Equation 1, we get

$$
\begin{aligned}
& \frac{\partial \tilde{S}_{i}\left(x_{k}\right)}{\partial S_{0}\left(y_{j}\right)}=\alpha_{k j} \exp \left[-b g_{i}^{T} \exp \left(L\left(y_{j}\right)\right) g_{i}\right] \\
& \frac{\partial \tilde{S}_{i}\left(x_{k}\right)}{\partial L\left(y_{j}\right)}=-b \alpha_{k j} S_{0}\left(y_{j}\right) \exp \left[-b g_{i}^{T} \exp \left(L\left(y_{j}\right)\right) g_{i}\right] \partial_{G_{i}}\left[\exp \left(L\left(y_{j}\right)\right)\right]
\end{aligned}
$$

where $\partial_{G_{i}}\left[\exp \left(L\left(y_{j}\right)\right)\right]=\partial\left[g_{i}^{T} \exp \left(L\left(y_{j}\right)\right) g_{i}\right] / \partial L\left(y_{j}\right)$ is the directional derivative of the matrix exponential. A detailed implementation for computing $\partial_{G_{i}}\left[\exp \left(L\left(y_{j}\right)\right)\right]$ is available in [8]. In the following sections, we discuss the MLE with Gaussian noise model followed by brief implementation details.

MLE with Gaussian Noise Assuming Gaussian noise of variance $\sigma^{2}$ on the LR image, the pdf of the observed signal $\tilde{S}_{i}$ knowing the expected signal $S_{i}$ is $p\left(\tilde{S}_{i} \mid S_{i}\right)$. Using Equation $3, r\left(\tilde{S}_{i}\left(x_{k}\right) \mid S_{i}\left(x_{k}\right)\right)$ can be computed as,

$$
p\left(\tilde{S}_{i} \mid S_{i}\right)=\frac{1}{\sigma \sqrt{2 \pi}} \exp \left(-\frac{\left(\tilde{S}_{i}-S_{i}\right)^{2}}{2 \sigma^{2}}\right) ; \quad r\left(\tilde{S}_{i} \mid S_{i}\right)=\frac{S_{i}-\tilde{S}_{i}}{\sigma^{2}},
$$

Alternatively, the MLE with Rician noise can be computed as in [8].

\subsection{Non-Stationary Spatial Prior}

Without any additional constraints, many HR tensor images could explain the observed low resolution DWIs. Among all these solutions, anisotropic regularization promotes smooth images in homogeneous areas while respecting the 
edges. The anisotropic behavior is achieved using a $\phi$-functional, i.e., $\operatorname{Reg}(s)=$ $\int_{\Omega} \phi(\|\nabla s\|)$, where $s$ is the intensity (scalar or tensor) of the voxel of image $\Omega$. In our implementation, we use $\phi(s)=2\left(1+s^{2} / \kappa^{2}\right)-2$, with $\kappa$ as the image normalization factor [8]. The gradient of $\operatorname{Reg}(s)$ is

$$
\nabla \operatorname{Reg}(s)=-2 \psi(|| \nabla s||) \triangle s-2 \nabla^{T}(\psi(|| \nabla s||)) \nabla s,
$$

where $\psi(s)=\phi^{\prime}(s) / s$. The anisotropic regularization can also be viewed as a spatial Markov random field (MRF) where the state (intensity) of the voxel is dependent on the states of the neighboring voxels. The anisotropic smoothing of posterior probabilities is equivalent to the Maximum A Posteriori (MAP) solution of a discrete MRF, making the full criterion in Equation 2 a MAP estimator.

The proposed HR tensor estimation method is similar to the one proposed in [8], in terms of the variational formulation. However, the total energy $\mathrm{E}($.$) is$ constrained through the signal degradation model in Equation 1. The novelty of the method lies in the fact that it estimates the tensors in HR while taking into account the unavoidable partial volume effects during acquisition, to produce a spatially coherent tensor field.

\subsection{Implementation}

The total energy $\mathrm{E}\left(\mathrm{S}_{0}, \mathrm{~L}\right)$ is minimized using the steepest descent method with line search algorithm.

$S_{0}{ }^{t+1}=S_{0}{ }^{t}-\frac{d t_{s}}{2}\left[\frac{\partial \operatorname{Sim}}{\partial S_{0}}+\lambda_{s} \nabla \operatorname{Reg}\left(\mathrm{S}_{0}\right)\right] ; \quad L^{t+1}=L^{t}-\frac{d t_{L}}{2}\left[\frac{\partial \operatorname{Sim}}{\partial L}+\lambda_{L} \nabla \operatorname{Reg}(\mathrm{L})\right]$,

where $d t_{s}$ and $d t_{L}$ are the step sizes for steepest descent. The two images $S_{0}$ and $L$ are optimized alternately, until convergence. The step-sizes are reduced by half if the the total variational energy is not decreased, until the step-size are too small $\left(d t_{s}<10^{-6}, d t_{L}<10^{-10}\right)$, that is the total energy $\mathrm{E}$ cannot be minimized any further. The algorithm is implemented in $\mathrm{C}++$.

\section{Comparison between Tensor Resampling and HR Tensor Estimation}

\subsection{Simulated LR acquisition}

Data were acquired using a GE $1.5 \mathrm{~T}$ scanner, with 24 encoding gradient directions (with a b-value of $700 \mathrm{~s} / \mathrm{mm}^{2}$ ). The image has $256 \times 256 \times 26$ voxels of size $0.9375 \times 0.9375 \times 5.5 \mathrm{~mm}^{3}$. The images are downsampled by a factor of two in the axial plane and the tensor field is estimated. This LR tensor field is then resampled to the original image size using Log-Euclidean interpolation [9]. The HR tensor estimation is used to estimate tensors at the original resolution from the downsampled images. In Fig. 1, the fractional anisotropy (FA) maps from both tensor resampling and HR estimation methods are shown. The FA map computed from the HR tensor estimation is better contrasted and less blurry when compared with the one computed from resampling the LR tensor image. 

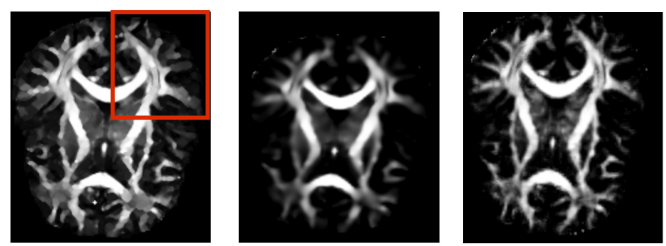

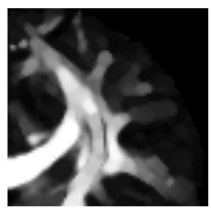

Ground Truth

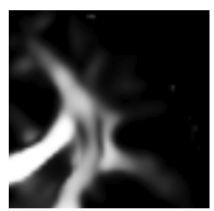

Resampling

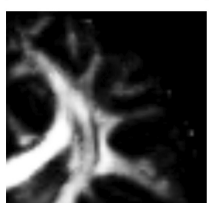

HR Tensor Estimation

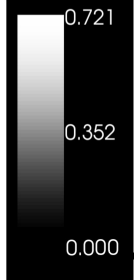

Fig. 1. Simulated LR acquisition. Top row: axial views of the ground truth image, FA computed from resampled tensor field and HR tensor estimation. The FA map computed from the HR tensor estimation method is better contrasted than the one computed from the resampled tensor field. Bottom row: zoomed region (red square).

\subsection{Increase in FA on real data}

In this section, we use a real dataset acquired with a Siemens $1.5 \mathrm{~T}$ scanner, with 21 encoding gradient directions and a b-value of $1000 \mathrm{~s} / \mathrm{mm}^{2}$. The images have $80 \times 80 \times 40$ voxels of size $3 \times 3 \times 3.3 \mathrm{~mm}^{3}$. The tensor field is estimated at the native resolution and is resampled using the Log-Euclidean framework. Several WM tracts which were not visible in the resampled images can easily be seen in the FA map, when the FA is computed from HR tensor estimation method. The HR tensor estimation algorithm presented in this paper shows an increase in FA values in the white matter regions. In Fig. 2, the FA maps are overlaid on the corresponding B0 image for better anatomical reference. In both the axial and coronal views, the external capsule and the corpus callosum can be clearly delineated with the HR tensor estimation method. We observed an increase in FA values by $43 \%$ in the WM regions (regions marked with arrows in Fig. 2) with the proposed method.

\subsection{Influence on Tractography: Quantitative Evaluation}

The same data set as in section 3.1 is used for a fiber tracking experiment. However, in this case the HR tensor estimation is done at $1 \mathrm{~mm}$ isotropic resolution and the tensor field is resampled to the same isotropic resolution. The fiber-tracking is done using the MedINRIA (www.med.inria.fr) 1.90 DTI-Track tool. All the voxels with FA $>0.25$, are considered as seed voxels for fiber tracking. The proposed method shows a statistically significant increase in the fiber lengths, when compared with tensor resampling. A one tailed t-test on fiber lengths gives a p-value less than $2 \times 10^{-16}$. The increase in FA values in WM regions as described in section 3.2 leads to an $82 \%$ increase in the number of 
seed voxels for HR tensor estimation and thus denser fibers. The fornix of the brain is tracked and the results are compared in Fig. 3 for tensor resampling (left column) and HR tensor estimation (middle column). There is a $25 \%$ increase in the mean length of fibers with the proposed tensor estimation method compared to tensor resampling. The right column in Fig. 3 shows the histogram for fiber length distribution.

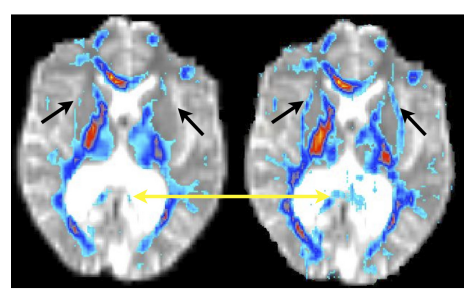

Interpolation

Super-resolution

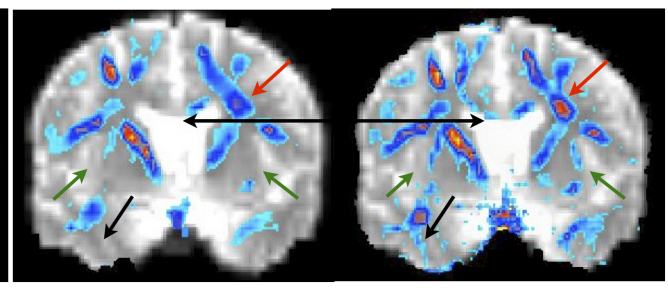

0.1

Fig. 2. Influence on FA in real clinical data: The axial and coronal views show that the external capsule and part of corpus callosum can be delineated using the HR tensor estimation. The corresponding arrows show regions with considerable increase in FA.

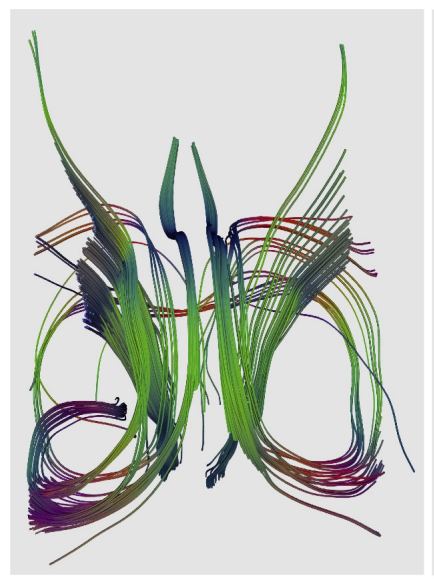

Resampling

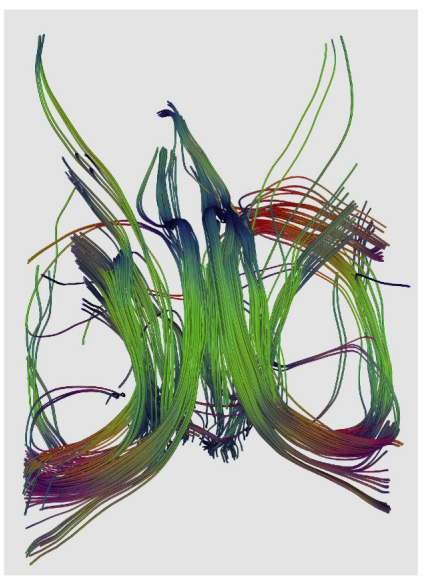

HR Tensor Estimation

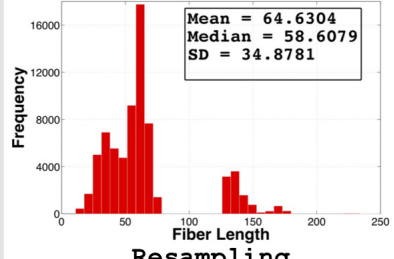

Resampling

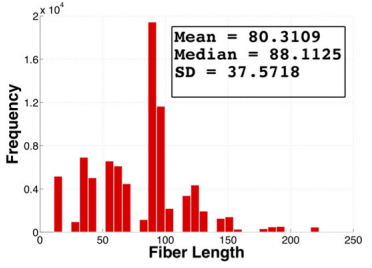

HR Tensor Estimation

Fig. 3. Middle column shows comparatively denser fiber bundle in the fornix region for the HR tensor estimation method (superior-inferior view) compared to tensor resampling (left column). Right column: a quantitative comparison of fiber lengths. 


\section{Conclusions}

In this paper, we tackled the problem for resampling low resolution DTI data on higher resolution for tractography or statistical analysis purposes at the population level. We propose to replace the resampling step by a DTI reconstruction at the high resolution using a MAP estimator with a spatial prior. The method is compared with tensor resampling method on simulated low resolution data as well as on real clinical data. Results showed better contrasted and less blurry FA maps on the simulated data. We confirmed our results with experiments on the Fiber Cup [10] which shows a $6.39 \%$ reduction in mean error in the principal diffusion direction. On the real data, a considerable increase in FA is observed, making some WM regions in parts of corpus callosum and external capsule recognizable. Fiber tracking with the HR tensor estimation shows statistically significant increase in the length and number of fibers. In the future, we would like to investigate if a better statistical power can be achieved in population based studies using the proposed method.

Acknowledgements We would like to acknowledge Nice University Hospital $(\mathrm{CHU})$ and Dr. Jean-Jacques Lemaire from Clermont-Ferrand Hospital (CHU) for providing DTI data. The work was partially supported by the European Research Council through the ERC Advanced Grant MedYMA 2011-291080 and by the French ANR Karametria project.

\section{References}

1. Vos, S.B., Jones, D.K., Viergever, M.A., Leemans, A.: Partial volume effect as a hidden covariate in DTI analyses. Neuroimage 55(4) (2011) 1566-1576

2. Peled, S., Yeshurun, Y.: Superresolution in MRI: Application to human white matter fiber tract visualization by diffusion tensor imaging. Magnetic Resonance in Medicene 45(1) (2001) 29-35

3. Greenspan, H., Oz, G., Kiryati, N., Peled, S.: MRI inter-slice reconstruction using super-resolution. Magnetic Resonance Imaging 20(5) (2002) 437-446

4. Irani, M., Peleg, S.: Motion analysis for image enhancement: Resolution, occlusion, and transparency. J. of Vis. Com. and Image Rep. 4(4) (1993) 1993-12

5. Scherrer, B., Gholipour, A., Warfield, S.K.: Super-resolution reconstruction to increase the spatial resolution of diffusion weighted images from orthogonal anisotropic acquisitions. Medical Image Analysis 16(7) (2012) 1465-1476

6. Nedjati-Gilani, S., Alexander, D.C., Parker, G.J.: Regularized super-resolution for diffusion MRI. In: Proc. of ISBI, IEEE (2008) 875-878

7. Stejskal, E., Tanner, J.: Spin diffusion measurements: Spin echoes in the presence of a time-dependent field gradient. J. of Chem. Phys. 42 (1965) 288

8. Fillard, P., Pennec, X., Arsigny, V., Ayache, N.: Clinical DT-MRI estimation, smoothing, and fiber tracking with Log-Euclidean metrics. IEEE Transanctions on Medical Imaging 26(11) (2007) 1472-1482

9. Arsigny, V., Fillard, P., Pennec, X., Ayache, N.: Log-Euclidean metrics for fast and simple calculus on diffusion tensors. Mag. Res. in Med. 56(2) (2006) 411-421

10. Fillard, P., Descoteaux, M., et. al: Quantitative evaluation of 10 tractography algorithms on a realistic diffusion MR phantom. Neuroimage 56(1) (2011) 220234 